\title{
Probing the cosmic microwave background temperature using the Sunyaev-Zeldovich effect
}

\author{
C. Horellou, M. Nord ${ }^{\star}$, D. Johansson, and A. Lévy
}

\author{
Onsala Space Observatory, Chalmers University of Technology, 43992 Onsala, Sweden \\ e-mail: horellou@oso.chalmers.se
}

Received 18 March 2005 / Accepted 4 July 2005

\begin{abstract}
We discuss the possibility of constraining the relation between redshift and temperature of the cosmic microwave background (CMB) using multifrequency Sunyaev-Zeldovich (SZ) observations. We have simulated a catalog of clusters of galaxies detected through their SZ signature assuming the sensitivities that will be achieved by the Planck satellite at 100, 143 and $353 \mathrm{GHz}$, taking into account the instrumental noise and the contamination from the Cosmic Infrared Background and from unresolved radiosources. We have parametrized the cosmological temperature-redshift law as $T \propto(1+z)^{(1-a)}$. Using two sets of SZ flux density ratios $(100 / 143 \mathrm{GHz}$, which is most sensitive to the parametrization of the $T-z$ law, and $143 / 353 \mathrm{GHz}$, which is most sensitive to the peculiar velocities of the clusters) we show that it is possible to recover the $T-z$ law assuming that the temperatures and redshifts of the clusters are known. From a simulated catalog of $\sim 1200$ clusters, the parameter $a$ can be recovered to an accuracy of $10^{-2}$. Sensitive SZ observations thus appear as a potentially useful tool to test the standard law. Most cosmological models predict a linear variation of the CMB temperature with redshift. The discovery of an alternative law would have profound implications on the cosmological model, implying creation of energy in a manner that would still maintain the black-body shape of the CMB spectrum at redshift zero.
\end{abstract}

Key words. large-scale structure of Universe - galaxies: clusters: general - cosmic microwave background

\section{Introduction}

One of the strongest predictions of the standard cosmological model is the linear variation of the Cosmic Microwave Background $(\mathrm{CMB})$ temperature with redshift: $T_{\mathrm{CMB}}(z)=$ $T_{0}(1+z)$ where $T_{0}$ is the temperature of the CMB at redshift zero. $T_{0}$ has been determined with a high accuracy from data taken by the Far Infrared Absolute Spectrometer (FIRAS) onboard the COBE satellite: $T_{0}=2.725 \pm 0.002 \mathrm{~K}$ (Mather et al. 1999). Those measurements do not imply that the CMB spectrum is Planckian at all redshifts, nor give information about how the temperature varies with redshift. Alternative cosmological models imply a different redshift dependence of the CMB temperature, for instance:

$T=T_{0}(1+z)^{(1-a)}$ with $0 \leq a \leq 1$

(Lima et al. 2000). Equation (1) requires energy injection into the $\mathrm{CMB}$, which could come from decay of vacuum energy (Freese et al. 1987). In such a case, the Planckian form of the radiation spectrum is preserved if the creation of photons is adiabatic, in the sense that the entropy per photon remains constant (Lima 1996).

^ Current address: Radioastronomisches Institut der Universität Bonn, Auf dem Hügel 71, 53121 Bonn, Germany.
An important test of such models would be to determine the temperature of the $\mathrm{CMB}$ at different redshifts through observations.

Shortly after the discovery of the CMB by Penzias \& Wilson (1965) it was realized that the relic radiation had already been indirectly observed through observations of interstellar absorption lines with very low excitation temperatures (see Thaddeus 1972, for a review of early observations). More recently, a temperature $T_{0}=2.729_{-0.031}^{+0.023} \mathrm{~K}$ has been derived from a survey to determine the amount of $\mathrm{CN}$ rotational excitation in interstellar clouds (Roth et al. 1993). Bahcall \& Wolf (1968) pointed out that the temperature of the CMB could be probed at much higher redshifts by studying the absorption spectra of quasars and searching for the species with energy states whose populations are sensitive to the ambient radiation temperature. This method, however, gives only an upper limit on the CMB temperature unless the local contribution to the excitation can be estimated, since other processes can contribute to the excitation of the atoms/molecules (Ge et al. 1997; Srianand et al. 2000; Molaro et al. 2002). So far, the standard law is consistent with observations, but so are alternative laws with $a=0.003 \pm 0.13$, or $b=0.99 \pm 0.22$ for a straight line $T(z)=T_{0}(1+b z)($ Losecco et al. 2001; see also Puy 2004).

Another way to probe the temperature of the CMB is provided by the Sunyaev-Zeldovich (SZ) effect (Zeldovich \& Sunyaev 1969; Sunyaev \& Zeldovich 1972). The CMB 


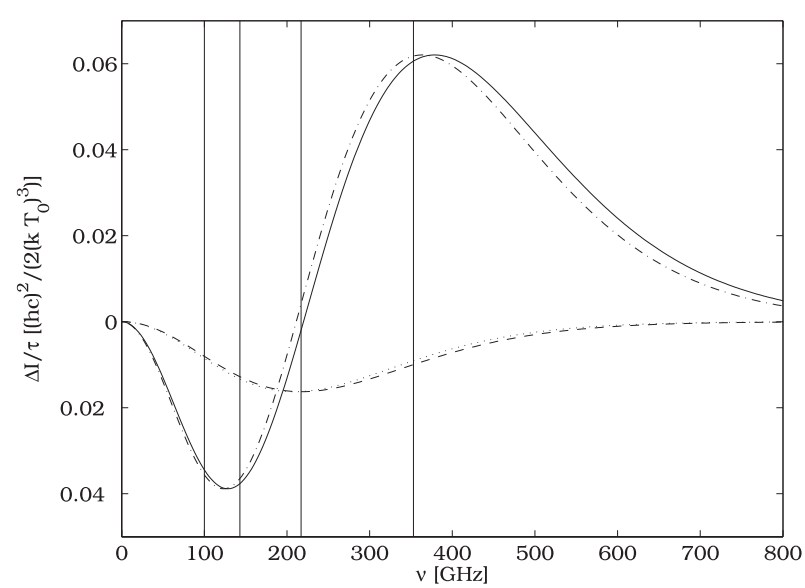

Fig. 1. Spectral signature of both the thermal SZ effect (solid line) and the kinetic effect (dashed line) for the standard cosmological temperature law $(a=0)$. The dash-dotted and the dotted lines show the corresponding curves for $a=0.1$ and $z=0.5$. The Planck frequencies used in this study are indicated. The following cluster parameters were used: $k T_{\mathrm{e}}=5 \mathrm{keV}, v_{\mathrm{r}}=+1000 \mathrm{~km} \mathrm{~s}^{-1}$.

spectrum is distorted due to interaction with intervening hot electrons. Massive clusters of galaxies contain significant amounts of hot gas that can produce a measurable SZ signal (see Fig. 1). Both the decrement at centimeter wavelengths and the increment in the millimeter range have been detected and large dedicated SZ surveys are planned (see Rephaeli 1995; Birkinshaw 1999; Carlstrom et al. 2002, for reviews). Using the standard scaling $v=v_{0}(1+z)$ and the temperature-redshift relation given in Eq. (1), the dimensionless frequency $x \equiv h v / k T$ depends on $z$, and we have

$x \equiv \frac{h v}{k T}=\frac{h v_{0}}{k T_{0}}(1+z)^{a}$,

where $h$ is the Planck constant and $k$ is the Boltzmann constant. The redshift independence of the SZ effect therefore occurs only when the standard temperature law is assumed.

Two methods can provide information on the CMB temperature at the redshift of the intervening cluster (Fabbri et al. 1978; Rephaeli 1980). The first one involves a precise measurement of the null point of the SZ signal, located around $218 \mathrm{GHz}$. The precise frequency depends both on the temperature of the hot gas and its redshift (see Sect. 3). It also depends on the peculiar velocity of the cluster. The second method involves measurement of the SZ signal at several frequencies; the temperature of the $\mathrm{CMB}$ can be derived from the ratio of the observed SZ flux densities in different bands, which for some frequencies depends weakly on the properties of the intracluster gas (see Sect. 4).

In this paper, we investigate the possibility of applying these methods to upcoming SZ observations such as those planned in the Planck mission (see Table 1, and Lamarre et al. 2003; Tauber 2004; Mennella et al. 2004, about this mission).
Table 1. Four frequency channels of the Planck high-frequency instrument relevant to this paper. The effective beam area has been calculated assuming a Gaussian beam. The levels of contamination due to the Cosmic Infrared Background and to unresolved radiosources are taken from Aghanim et al. (2005).

\begin{tabular}{lllcc}
\hline \hline Central frequency (GHz) & 100 & 143 & 217 & 353 \\
Bandwidth (GHz) & 33 & 47 & 72 & 116 \\
Angular resolution (arcmin) & 9.2 & 7.1 & 5.0 & 5.0 \\
Effective beam area (arcmin $\left.{ }^{2}\right)$ & 95.9 & 57.1 & 28.3 & 28.3 \\
& & & & \\
Random noise (mJy/beam) & 14.0 & 10.2 & 14.3 & 27.0 \\
Cosmic Infrared Background (mJy/beam) & 10.5 & 15.6 & 23.7 & 81.9 \\
Unresolved radiosources (mJy/beam) & 26.0 & 15.5 & 8.6 & - \\
Total uncertainty (mJy/beam) & 31.4 & 24.3 & 29.0 & 86.3 \\
\hline
\end{tabular}

\section{The SZ effect}

The thermal SZ effect can be described in terms of the Compton $y$ parameter:

$y=\sigma_{\mathrm{T}} \int n_{\mathrm{e}}\left(\frac{k T_{\mathrm{e}}}{m_{\mathrm{e}} c^{2}}\right) \mathrm{d} l$

where $\sigma_{\mathrm{T}}=6.65 \times 10^{-25} \mathrm{~cm}^{2}$ is the Thomson cross section, $n_{\mathrm{e}}$ is the number density of electrons, $T_{\mathrm{e}}$ is the temperature of the intracluster gas, $m_{\mathrm{e}}$ is the electron mass, and $c$ is the speed of light. The $y$ parameter is a measure of the integrated pressure of the intracluster gas along the line-of-sight.

Due to inverse Compton scattering off the hot electrons, the spectrum of the CMB is distorted. In the non-relativistic limit (if the velocity of the hot electrons is negligible with respect to $c$ ), the change in the CMB flux density is

$S_{v}(x)=S_{v}^{\mathrm{CMB}}(x) Q(x) Y$

where $S_{v}^{\mathrm{CMB}}=2(k T)^{3} x^{3} /\left((h c)^{2}\left(\mathrm{e}^{x}-1\right)\right)$ is the unperturbed CMB flux density. The spectral distortion of the incident CMB spectrum is described by the function

$Q(x)=\frac{x \mathrm{e}^{x}}{\mathrm{e}^{x}-1}\left(\frac{x}{\tanh (x / 2)}-4\right)$.

$Y$ is the integrated Compton parameter over the projected area of the cluster on the sky:

$Y=D_{\mathrm{A}}^{-2} \int y \mathrm{~d} A$,

where $D_{\mathrm{A}}$ is the angular diameter distance.

For high electron temperatures the relativistic effect has to be taken into account (e.g., Wright 1979; Fabbri 1981; Rephaeli \& Yankovitch 1997). Useful numerical tables and analytic fitting formulae have been provided by several authors (see Itoh \& Nozawa 2004, and references therein).

The kinetic SZ effect is due to the motion of the cluster with respect to the $\mathrm{CMB}$ frame:

$\frac{\Delta T}{T}=-\tau \frac{v_{\mathrm{r}}}{c}$,

with the convention that $v_{\mathrm{r}}>0$ for a receding cluster. 


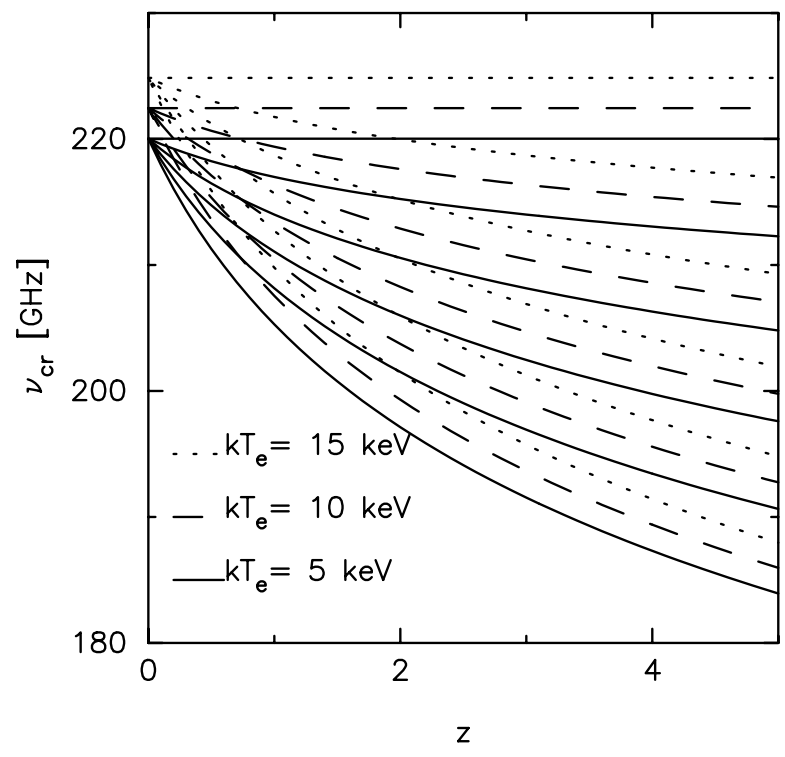

Fig. 2. Variation of the cross-over frequency as a function of the redshift of the intervening cluster for different cluster temperatures and different $T-z$ relations (the parameter $a$ varies from 0 to 0.1 by 0.02 from top to bottom). Here the peculiar velocity of the clusters has been taken as equal to 0 .

\section{Measurement of the cross-over frequency}

The location of the null point of the SZ signal $v_{\mathrm{cr}}$ can give information on the temperature of the $\mathrm{CMB}$ at the redshift of the intervening cluster if it doesn't obey the standard law. From Eq. (2) we have $v_{\mathrm{cr}}=x_{\mathrm{cr}} \frac{k T_{0}}{h}(1+z)^{-a}$. Sazanov \& Sunyaev (1998a,b) provide a simple approximation for the nondimensional cross-over frequency $x_{\mathrm{cr}}$ :

$x_{\mathrm{cr}}=3.830\left(1+0.30 \frac{v_{\mathrm{r}}}{c} \frac{m_{\mathrm{e}} c^{2}}{k T_{\mathrm{e}}}+1.1 \frac{k T_{\mathrm{e}}}{m_{\mathrm{e}} c^{2}}+1.5 \frac{v_{\mathrm{r}}}{c}\right)$

where $v_{r}$ is the velocity of the cluster along the line of sight and $T_{\mathrm{e}}$ is the temperature of the intracluster gas. For $v_{r}=0$ one recovers the approximation given by Fabbri (1981). Itoh et al. (1998) provide a second-order fit to the numerical result, $x_{\mathrm{cr}}=$ $3.83\left(1+1.1674 \theta_{\mathrm{e}}-0.8533 \theta_{\mathrm{e}}^{2}\right)$, valid for $0<k T_{\mathrm{e}}<50 \mathrm{keV}$ and more accurate at high $T_{\mathrm{e}}$ than Eq. (8), and where $\theta_{\mathrm{e}}=$ $k T_{\mathrm{e}} / m_{\mathrm{e}} c^{2}$. In the following we use that approximation in the figures where $v_{\mathrm{r}}=0$.

Figure 2 shows the variation of the cross-over frequency as a function of the redshift of the intervening cluster for different cluster temperatures and different $T-z$ relations. Here we first consider $v_{\mathrm{r}}=0$. For $a=0$, one recovers the known result that $v_{\mathrm{cr}}$ is constant with redshift and increases with increasing $T_{\mathrm{e}}$. For different values of $a$, the curves are split and $v_{\mathrm{cr}}$ decreases with increasing $a$. There is thus a parameter degeneracy between $T_{\mathrm{e}}$ and $a$.

Figure 3 illustrates the dependence on the peculiar velocity of the cluster, $v_{\mathrm{r}}$, introducing a further degeneracy.

Those parameter degeneracies and the large bandwidths of current bolometers make it difficult to apply this method in practice to constrain the $\mathrm{CMB}$ temperature.

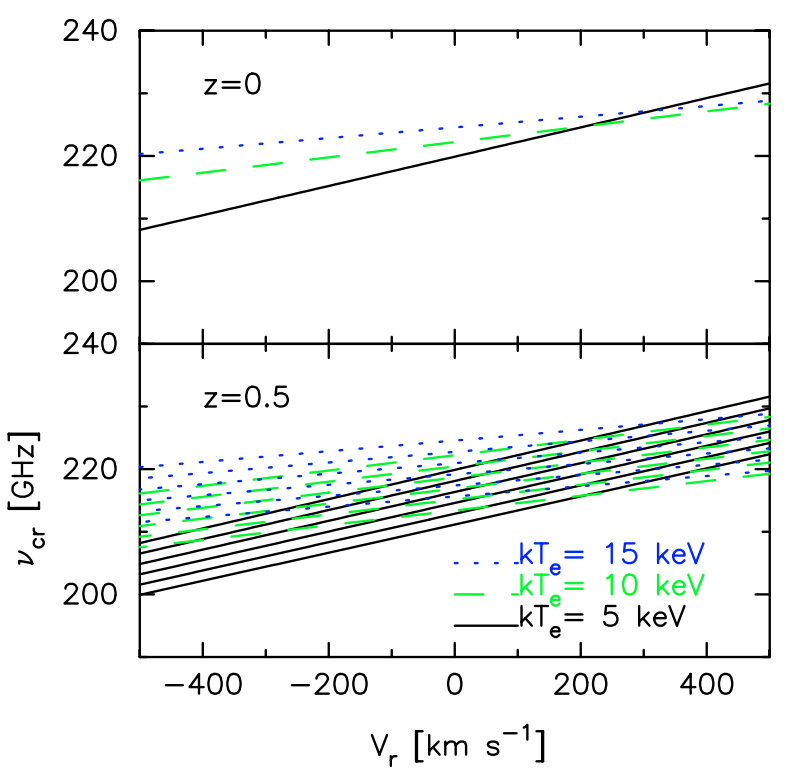

Fig. 3. Variation of the cross-over frequency as a function of the peculiar velocity of the intervening cluster for different cluster temperatures and different $T-z$ relations (the parameter $a$ varies from 0 to 0.1 by 0.02 from top to bottom).

\section{Measurements at different frequencies}

A more promising method consists of using ratios of observed flux densities in several frequency bands. It has recently been applied sucessfully to determine the CMB temperature at the redshift of the Coma cluster (at $z=0.0231$ ) and of Abell 2163 at $z=0.203$ by Battistelli et al. (2002) who used observations at 32,143 and $272 \mathrm{GHz}$.

We have calculated the flux density of the thermal SZ effect using the analytic fitting formulae given by Itoh et al. (1998) valid in the relativistic case. We have added the contribution of the kinetic effect. The flux density ratio depends weakly on the cluster temperature for frequencies between $32 \mathrm{GHz}$ and $\sim 170 \mathrm{GHz}$. If the second measurement $\left(v_{2}\right)$ is done at a higher frequency, the flux density ratio decreases with increasing $T_{\mathrm{e}}$, more strongly as $v_{2}$ increases. For different values of the $a$ parameter, the curves are split. The magnitude of the split increases with redshift. Figure 4 shows the variation of the flux density ratios with the cluster temperature, Fig. 5 the variation with the peculiar velocity of the cluster. Observations with new X-ray satellites (XMM, Chandra) make it possible to measure $T_{\mathrm{e}}$ with good accuracy. Note, however, that the flux density ratios depend only weakly on the cluster temperature. To constrain the parameter $a$, it would be necessary to constrain the peculiar velocity of a cluster independently. Alternatively, it is possible to constrain $a$ by observing a large number of clusters with known gas temperatures, as will be shown in the next section.

\section{Simulating a large SZ survey}

We seek to simulate the yield of a Planck-like SZ survey to test how effectively our parametrization of the $T-z$ relation can be constrained. One way to do this is to simulate sky maps 


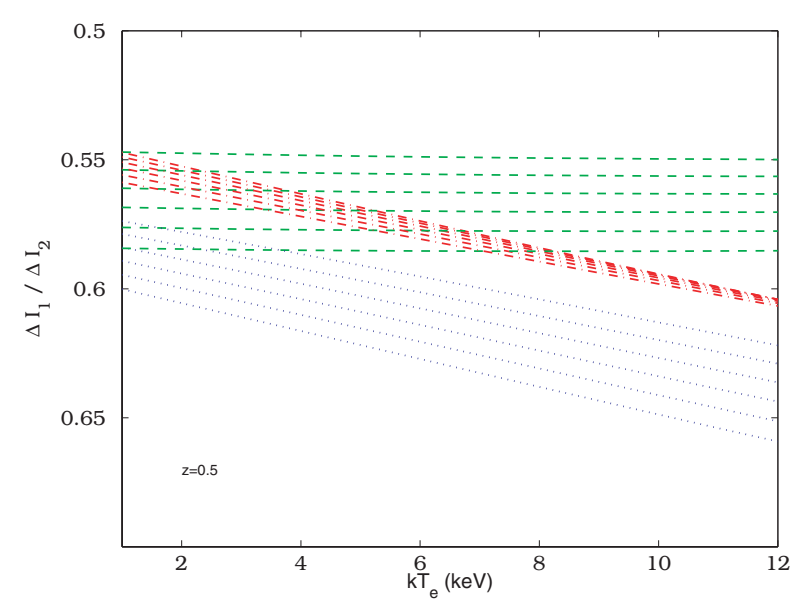

Fig. 4. Variation of the flux density ratio as a function of the cluster temperature for clusters at $z=0.5$ and different $z-T$ relations. The parameter $a$ varies from 0 to 0.1 by 0.02 from bottom to top. Here the peculiar velocities have been set to zero. The dashed lines show the $100 / 143 \mathrm{GHz}$ flux density ratio, multiplied by -0.6 for clarity. The dash-dotted lines show the $143 / 353 \mathrm{GHz}$ ratio. The dotted lines show the $100 / 353 \mathrm{GHz}$ ratio. Clearly, the $100 / 143 \mathrm{GHz}$ ratio is most sensitive to $a$ and varies least with the cluster temperatures.

based on $N$-body simulations with $\mathrm{SZ}$ signals as well as random and systematic noise in the relevant frequency bands and then use a suitable cluster extraction algorithm (see, for example, Geisbüsch et al. 2005). Here we take the simpler approach of directly simulating a cluster catalog from the redshift and mass distribution of dark matter halos and computing the SZ signals assuming that the clusters are unresolved. This method is justified for this study as it gives approximately the same number of recovered clusters as more general methods.

\subsection{The model}

We have used a fiducial $\Lambda$ CDM cosmology with $\Omega_{0}=0.3$, $\lambda_{0}=0.7, H_{0}=100 h \mathrm{~km} \mathrm{~s}^{-1} \mathrm{Mpc}^{-1}, h=0.7, \sigma_{8}=0.9$, consistent with the first-year WMAP results (Spergel et al. 2003). We have used the Sheth \& Tormen (1999) cluster mass function, which provides an excellent match to the results from large numerical simulations, and has the advantage of having a theoretical justification in terms of the ellipsoidal collapse of clusters (Sheth et al. 2001). The mass function can be expressed as (Mo \& White 2002):

$n(M, z) \mathrm{d} M=A\left(1+\frac{1}{v^{\prime 2 q}}\right) \sqrt{\frac{2}{\pi}} \frac{\rho_{0}}{M} \frac{\mathrm{d} v^{\prime}}{\mathrm{d} M} \mathrm{e}^{\left(-\frac{v^{\prime 2}}{2}\right)} \mathrm{d} M$

where $\rho_{0}$ is the comoving background density, $v^{\prime}=\sqrt{\alpha} v$, $\alpha=0.707, A \simeq 0.322, q=0.3, v=v(M, z)=\delta_{\mathrm{c}} /(D(z) \sigma(M))$, $\delta_{\mathrm{c}}$ is the linear overdensity of a perturbation at the time of collapse and virialization; $\delta_{\mathrm{c}}$ varies weakly with the cosmological parameters and has a value of $\delta_{\mathrm{c}} \simeq 1.69 . \sigma(M, z)$ is the variance of the fluctuation spectrum on a mass scale $M=\frac{4}{3} \pi R^{3} \rho_{0}$.

The number density of clusters above a certain mass per unit steradian per redshift interval is

$\frac{\mathrm{d}^{2} N(z)}{\mathrm{d} \Omega \mathrm{d} z}=\frac{\mathrm{d}^{2} V_{\mathrm{c}}}{\mathrm{d} \Omega \mathrm{d} z} \int_{M_{\lim (z)}^{+\infty}}^{+\infty} n(M, z) \mathrm{d} M$

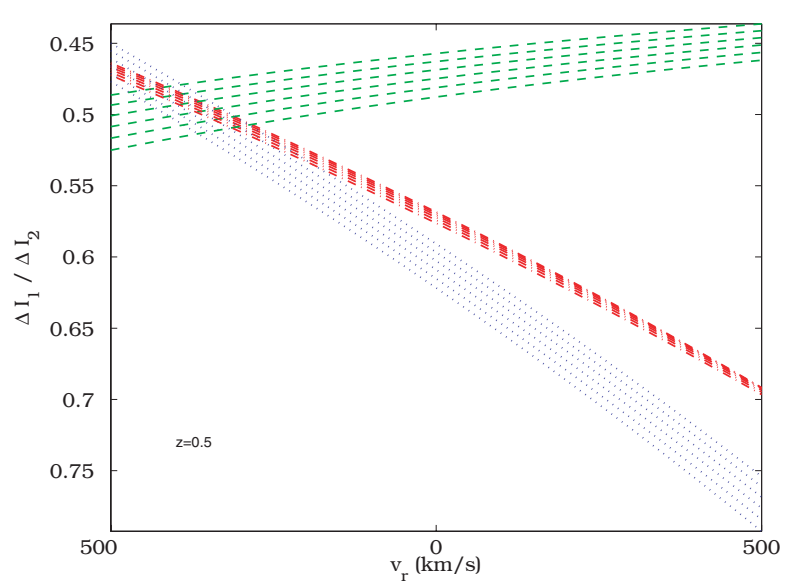

Fig. 5. Same as Fig. 4 but as a function of the peculiar velocity of the cluster. The 100/143 GHz flux density ratios have been multiplied by 0.5 for clarity. Here $k T_{\mathrm{e}}=5 \mathrm{keV}$. The $143 / 353 \mathrm{GHz}$ flux density ratio is most sensitive to the peculiar velocities.

where the comoving volume is

$\frac{\mathrm{d}^{2} V_{\mathrm{c}}}{\mathrm{d} \Omega \mathrm{d} z}=c \frac{D_{\mathrm{A}}^{2}(z)(1+z)^{2}}{H(z)}$,

where $D_{\mathrm{A}}$ is the angular diameter distance and $H(z)$ is the Hubble parameter.

A mass-temperature relation is needed in order to assign a gas temperature to a cluster. If the gas is in hydrostatic equilibrium with the gravitational potential of the cluster, then:

$k T_{\mathrm{e}}=\left|\frac{1}{\mathrm{~d} \ln \rho_{\mathrm{gas}(\mathrm{r})} /\left.\mathrm{d} \ln r\right|_{\mathrm{vir}}}\right| \mu m_{\mathrm{P}} \frac{G M_{\mathrm{vir}}}{r_{\mathrm{vir}}}$

where $\rho_{\text {gas }}$ is the gas density, $r_{\mathrm{vir}}$ is the virial radius, and $\mu=$ $4 /(5 X+3)$ is the mean molecular weight, $X$ is the hydrogen mass fraction and $m_{\mathrm{P}}$ is the mass of the proton.

We have adopted the Navarro et al. (1997) profile for the gas:

$\left.\frac{\mathrm{d} \ln \rho_{\mathrm{gas}}}{\mathrm{d} \ln r}\right|_{r_{\mathrm{vir}}}=-\frac{1+3 c}{1+c}$

with

$c=6\left(\frac{M_{\mathrm{vir}}}{10^{14} h^{-1} M_{\odot}}\right)^{-1 / 5}$.

Taking a constant $c=5$ yields an error in $\left.\frac{\mathrm{d} \ln \rho_{\mathrm{gas}}}{\mathrm{d} \ln r}\right|_{r_{\mathrm{vir}}}$ in Eq. (13) of less than $5 \%$ for the range of masses considered.

Introducing $\Delta_{c}$, the average density within the cluster relative to the critical density of the background at the redshift of the cluster, one obtains:

$$
\begin{aligned}
\frac{k T_{\mathrm{e}}}{\mathrm{keV}}= & 2\left|\frac{7.75}{\mathrm{~d} \ln \rho_{\mathrm{gas}(\mathrm{r})} /\left.\mathrm{d} \ln r\right|_{r_{\mathrm{vir}}}}\right| \\
& \times\left(\frac{6.8}{5 X+3}\right)\left(\frac{M_{\mathrm{vir}}}{10^{15} h^{-1} M_{\odot}}\right)^{2 / 3} \\
& \times(1+z)\left[\frac{\Omega_{0}}{\Omega(z)}\right]^{1 / 3}\left(\frac{\Delta_{\mathrm{c}}}{178}\right)^{1 / 3}
\end{aligned}
$$



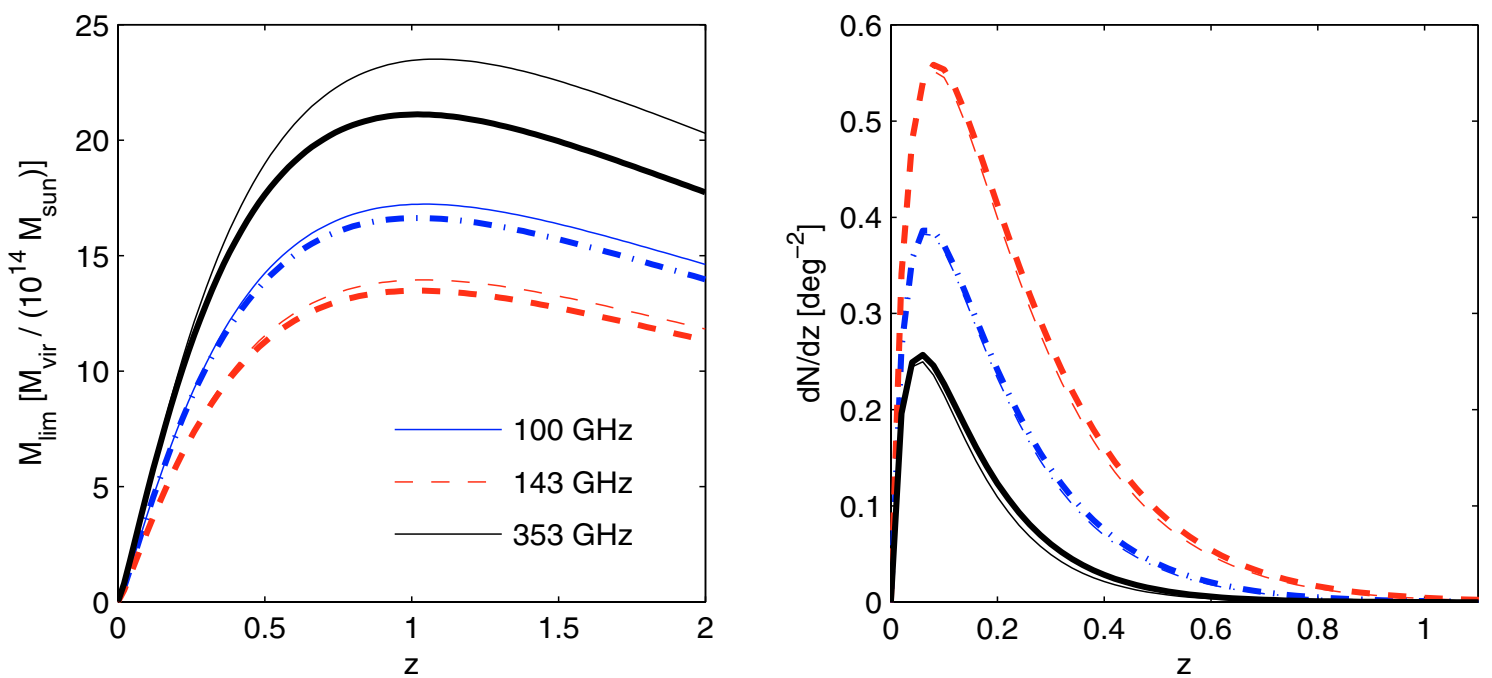

Fig. 6. Left: limiting mass as a function of redshift at three frequencies for observations at the $3 \sigma$ level using Planck, taking into account both the random instrumental noise and the levels of contamination due to the Cosmic Infrared Background and to unresolved radiosources given in Table 1. The thin lines correspond to the relativistic calculation and the thick lines to the non-relativistic one. The lowest curves correspond to $143 \mathrm{GHz}$, the intermediate ones to $100 \mathrm{GHz}$, and the highest ones to $353 \mathrm{GHz}$. Right: number density of clusters per redshift interval and per square degree on the sky as a function of redshift, above the limiting mass.

We have calculated $\Delta_{\mathrm{c}}$ from $\Delta_{\mathrm{vir}}=\rho / \rho_{\mathrm{bg}}$, taken from the fits given by Kitayama \& Suto (1996):

$\Delta_{\text {vir }} \approx 18 \pi^{2}\left(1+0.4093 w_{\mathrm{vir}}^{0.9052}\right)$,

where $w_{\mathrm{vir}}=1 / \Omega_{\mathrm{vir}}-1$, and $\Omega_{\mathrm{vir}}$ is the density parameter at virialisation; $\Omega_{\mathrm{vir}}=\frac{\Omega_{0}\left(1+z_{\mathrm{vir}}\right)^{3}}{\Omega_{0}\left(1+z_{\mathrm{vir}}\right)^{3}+\lambda_{0}}$ for a flat $\Lambda \mathrm{CDM}$ model.

We have added a spread of $30 \%$ to the mass-temperature relation.

As for the peculiar velocities of the clusters, we have drawn them from a Gaussian distribution with zero mean. Sheth \& Diaferio (2001) provide a fit for the dispersion as a function of the mass of the cluster:

$\sigma_{\text {halo }}(M)=\frac{\sigma_{\text {fit }}}{1+\left(R / R_{\text {fit }}\right)^{\eta}}$

with $R=\left(3 M / 4 \pi \rho_{0}\right)^{1 / 3}, \sigma_{\text {fit }}=414.7 \mathrm{~km} \mathrm{~s}^{-1}, R_{\mathrm{fit}}=$ $34.67 h^{-1} \mathrm{Mpc}$, and $\eta=0.85$ for a $\Lambda \mathrm{CDM}$ model with the same parameters as ours. $\sigma_{\text {halo }}^{2}$ is the dispersion of the Maxwellian distribution (three-dimensional peculiar velocity distribution). For the peculiar velocity along the line-of-sight, we thus took $\sigma_{\text {pec }}^{2}=\sigma_{\text {halo }}^{2} / 3$.

\subsection{The limiting mass}

The integration in Eq. (10) is to be done from a limiting mass which is redshift-dependent. The majority of the clusters will be unresolved at the resolution of the Planck instruments (Kay et al. 2001).

The SZ flux can be expressed as

$$
\begin{aligned}
S_{v}= & 2.29 \times 10^{4} \frac{x^{3}}{\mathrm{e}^{x}-1} Q(x) \times 1.70 \times 10^{-2} h\left(\frac{f_{\mathrm{ICM}}}{0.1}\right) \\
& \times\left(\frac{1+X}{1.76}\right)\left(\frac{D_{A}}{100 h^{-1} \mathrm{pc}}\right)^{-2}\left(\frac{k T_{\mathrm{e}}}{\mathrm{keV}}\right) \mathrm{mJy},
\end{aligned}
$$

where $f_{\mathrm{ICM}}$ is the gas mass fraction that we will take constant and equal to 0.1 and $k T_{\mathrm{e}}$ is taken from Eq. (15) (Fan \& Chiueh 2001).

We have calculated the limiting mass for Planck observations at a detection level of $3 \sigma$, where $\sigma$ is the total uncertainty given in Table 1. The error estimates on the flux densities are discussed in Sect. 5.4.

\subsection{The $S Z$ catalog}

We have distributed clusters in mass and redshift according to Eqs. (9) and (10), above the limiting mass discussed above (see Fig. 6). After excluding the nearby clusters at redshifts below 0.05 , we obtain a catalog of $\sim 1200$ clusters.

We have then calculated the flux density ratios for various frequency pairs and for different values of the parameter $a$ in the cosmological redshift-temperature law. We have used the bandwidths of the Planck instruments and calculated the observed SZ flux density assuming a top-hat response of the filters $\left(R=1\right.$ for $v_{\mathrm{c}}-\Delta v / 2<v<v_{\mathrm{c}}+\Delta v / 2$ and $R=0$ elsewhere (see Table 1)).

\subsection{Error estimates}

Errors are simulated through use of the noise levels quoted in Table 1. As for the uncertainties on the flux density ratios, we have propagated the uncertainties on the individual flux densities.

The random noise and the contribution from the cosmic infrared background and from unresolved radiosources have been taken from Aghanim et al. (2005) and added in quadrature. Dust emission has been neglected as the levels of contamination are orders of magnitude lower than for the other contaminants mentioned above. 

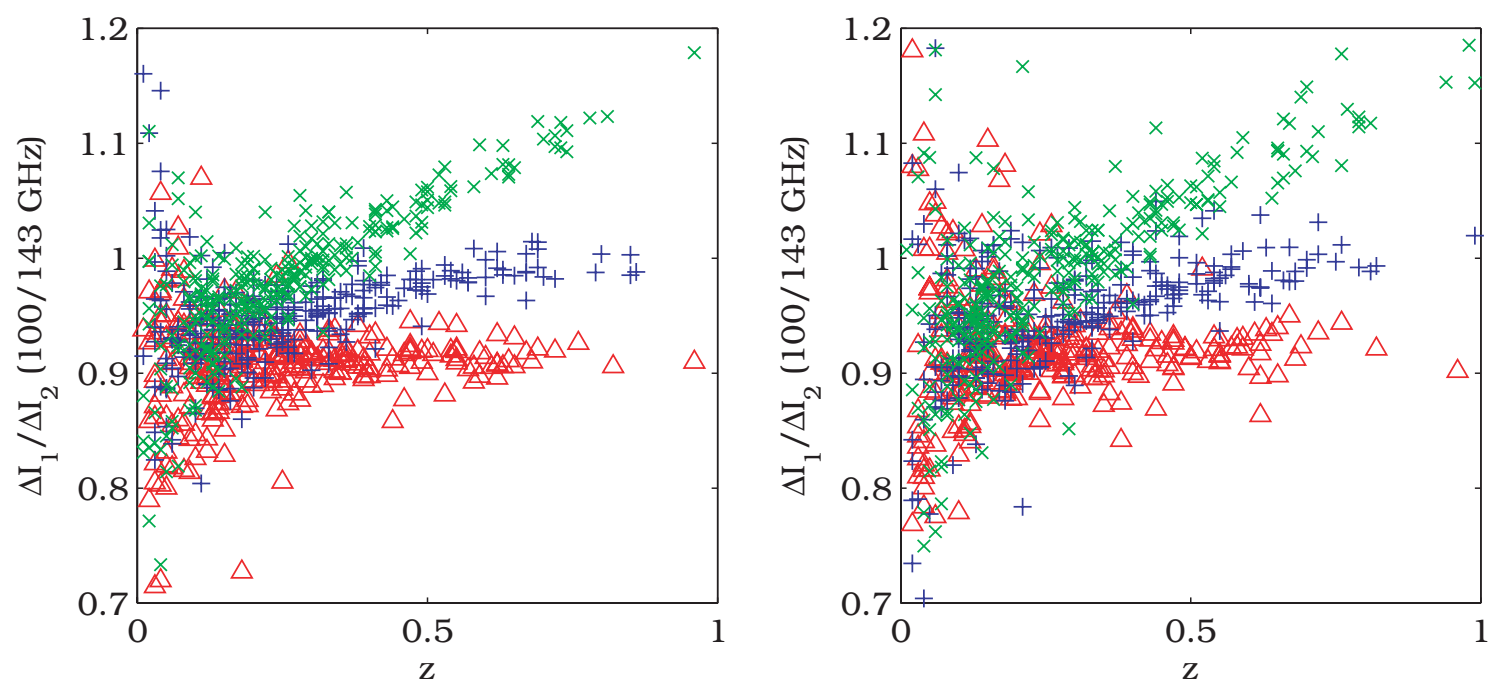

Fig. 7. Flux density ratio versus redshift for simulations with different values of the parameter $a$. For clarity, only instrumental noise has been included. The triangles correspond to $a=0$, the plusses to $a=0.1$, the crosses to $a=0.2$. Left: in the absence of peculiar motions $\left(v_{\mathrm{r}}=0\right)$. The distributions are clearly different. Right: including the peculiar motions. The spread in the flux density ratio is much larger than when $v_{\mathrm{r}}=0$, and the different $a$ distributions are mixed at low redshifts.

Another contaminating source is the primary temperature fluctuations of the CMB, which are important for observations with a large beam such as that of Planck. The primordial anisotropies have the same spectral signature as the kinetic SZ effect. Promising techniques to separate the primary CMB fluctuations from the kinetic SZ effect have been developed, for example by taking advantage of the spatial correlation between the thermal and the kinetic effects (see Forni \& Aghanim 2004, and references therein). In the present study, we have assumed that the contamination from the CMB as well as the influence of the motion of the Solar System (Chluba et al. 2005) could be removed. Observations with a smaller telescope beam close to the average size of the cluster (around $1 \mathrm{arcmin}$ ) wouldn't suffer significantly from contamination from the CMB. It has also been shown that such observations would provide an optimum survey yield for the detection of clusters (Battye \& Weller 2005).

Although we assume precise and unbiased knowledge of the electron temperatures in the catalog, it should be pointed out that the results are not very sensitive to the cluster temperatures, as shown in Fig. 4. Adding an uncertainty of up to $20 \%$ on the cluster temperatures doesn't affect the final result significantly. Errors due to peculiar velocities have to be modelled more carefully, however; not only because of the limited precision with which these can be extracted from the SZ data, but also because $\Delta I_{1} / \Delta I_{2}$ is more sensitive to $v_{\mathrm{r}}$ than to $k T_{\mathrm{e}}$.

We use a separate frequency ratio to fit to $v_{r}$ for each individual cluster. Error bars on each peculiar velocity are converted into error bars in the ratio used for the CMB temperature by simply calculating this ratio at the endpoints of the $68 \%$ confidence interval of the peculiar velocities. These systematic errrors are then added in quadrature to the other errors.

\subsection{Analysis}

As a first step, we have compared simulated SZ observations of catalogs with different $a$. Figure 7 shows the flux density ratio

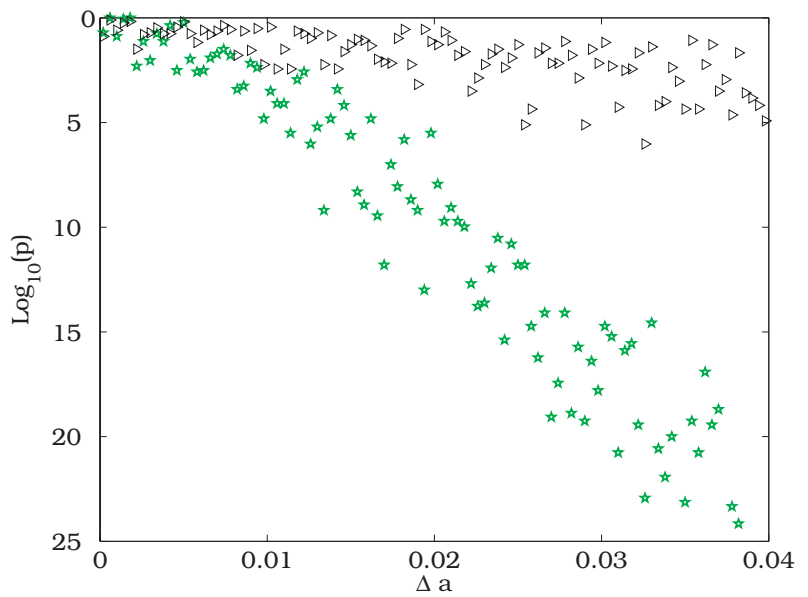

Fig. 8. Kolmogorov-Smirnov test: the significance level $p$ is shown as a function of $a . p$ is the probability that the two data sets are drawn from the same distribution. Stars show the results for the $100 / 143 \mathrm{GHz}$ frequency pair, triangles for the $143 / 353 \mathrm{GHz}$ pair. The $100 / 143 \mathrm{GHz}$ pair appears as the better one, as the probability that the $a=0$ distribution be the same as a one with $a \neq 0$ drops most rapidly with increasing $a$. Each data set contains 1000 clusters.

for the frequency pair $100 / 143 \mathrm{GHz}$ as a function of redshift in two cases: first, in a simulated catalog where all clusters have zero peculiar velocity (left), and then where peculiar velocities have been assigned according to Eq. (17). Clearly, the introduction of peculiar velocities confuses the picture and makes it more difficult to distinguish the distributions with different $a$. In order to compare quantitatively the distributions with the inclusion of peculiar velocities, we have run a KolmogorovSmirnov test (Fig. 8). We see clearly that the distributions are statistically different for a sufficiently large $a$.

This first test is encouraging. In practice, however, one would like to be able to determine the value of the $a$ parameter from one single simulation, since we have only one observable 

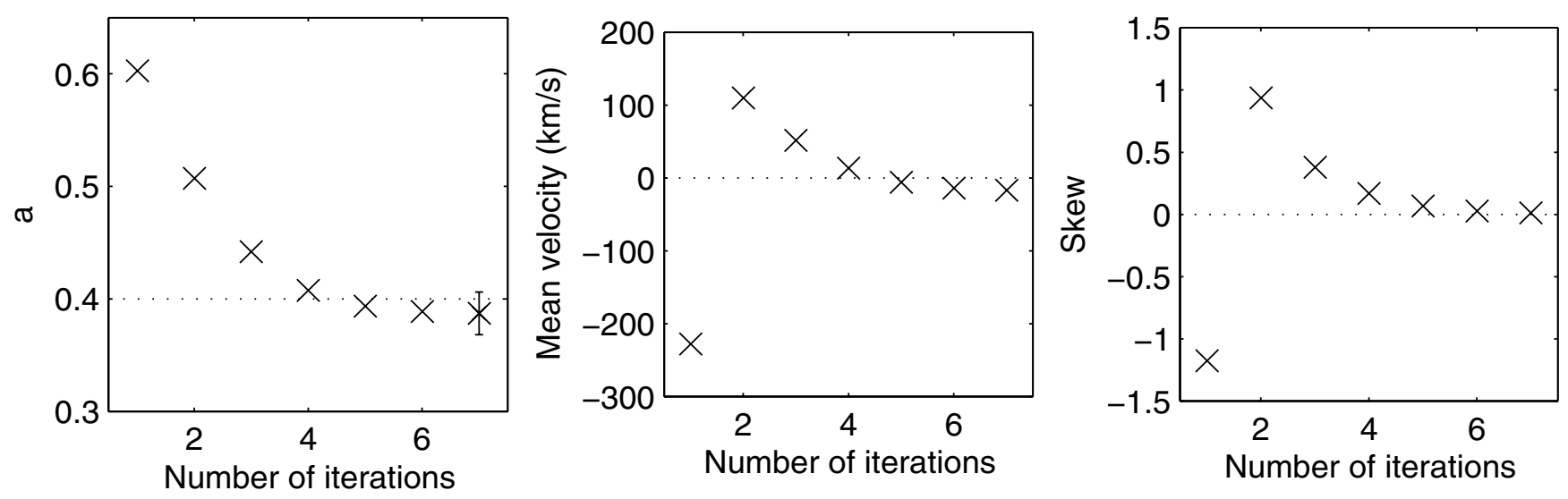

Fig. 9. Convergence of the method. Here a simulation with $a=0.4$ was performed. The method clearly converges toward that value of $a$, as can also be seen from several diagnositics: the mean value of the peculiar velocity distribution and the skewness converge toward zero as $a$ tends toward its original value.

universe at our disposal! To do this, we have devised the following iterative method:

1. First assuming $a=0$, we do a $\chi^{2}$ analysis to fit the peculiar velocities to the simulated flux density ratios at two frequencies, namely 143 and $353 \mathrm{GHz}$, which is the frequency pair which is most sensitive to peculiar velocities. We do this for each cluster.

2. Using those velocities, we then do a $\chi^{2}$ analysis to fit the whole catalog to $a$, now using the frequency pair $100 / 143 \mathrm{GHz}$, which is most sensitive to $a$.

3. Taking the parameter $a$ obtained at step 2, we iterate and fit to the peculiar velocities, and then fit to a new value of $a$.

We iterate until the method converges. Figure 9 shows clearly that it does: here we have deliberately used a large $a$ : 0.4 . After a few iterations, the method converges. We have also examined the distribution of the peculiar velocities at each iteration. At the first iteration (when $a$ is taken equal to zero), the distribution of peculiar velocities is clearly non-Gaussian, as indicated by its skewness. With each integration, the distribution of velocities converges toward the original one; the parameter $a$ also tends toward its original value. In order to test the robustness of the method, we repeated 200 times a simulation with $a=0$. The distribution of the inferred $a$ parameters follows a Gaussian, with a dispersion around $10^{-2}$.

\section{Conclusion}

We have examined the possibility to constrain the cosmological temperature-redshift law from multifrequency SZ observations. The advantage of using flux density ratios is to eliminate a possible dependence on cluster parameters, in particular the optical depth and, to some extent, the cluster temperature. After several tests, we have selected the $100 / 143 \mathrm{GHz}$ frequency pair as particularly sensitive to our parametrization of the $T-z$ law, and the $143 / 353 \mathrm{GHz}$ pair as sensitive to the cluster peculiar velocities. The iterative method that we have devised makes it possible to recover the parameter $a$ of the $T-z$ law from SZ flux density ratios of a large number of clusters. Our simulated catalog contains $\sim 1200$ clusters. We have assumed a
$\Lambda \mathrm{CDM}$ cosmology, a full-sky survey and detection at more than three times the expected sensitivity of the Planck instrument taking into acccount contamination by the Cosmic Infrared Background and radiosources. Note, however, that the method is independent of the cosmological parameters and could be applied to any catalog of clusters for which redshifts and, to some extent, temperatures are known.

Most cosmological models predict a linear variation of the CMB temperature with redshift. Sensitive SZ observations appear as a potentially useful tool to test the standard law. The discovery of an alternative law would have profound implications on the cosmological model, implying creation of energy in a manner that would still maintain the black-body shape of the CMB spectrum at redshift zero.

Acknowledgements. We are grateful to John Black for useful comments on the manuscript. C.H. is grateful to John Bahcall for an invitation to visit the Institute for Advanced Study in Princeton in September 2004 where part of this work was completed. C.H. acknowledges financial support from the Swedish Research Council Vetenskapsrådet.

\section{References}

Aghanim, N., Hansen, S. H., \& Lagache, G. 2005, A\&A, 439, 901 Bahcall, J. N., \& Wolf, R. A. 1968, ApJ, 152, 701

Battistelli, E. S., De Petris, M., Lamagna, L., et al. 2002, ApJ, 580, L101

Battye, R. A., \& Weller, J. 2005, MNRAS, 362, 171

Birkinshaw, M. 1999, Phys. Rep., 310, 97

Carlstrom, J. E., Holder, G. P., \& Reese, E. D. 2002, ARA\&A, 40, 643

Chluba, J., Hütsi, G., \& Sunyaev, R. A. 2005, A\&A, 434, 811

Fabbri, R. 1981, Ap\&SS, 77, 529

Fabbri, R., Melchiorri, F., \& Natale, V. 1978, Ap\&SS, 59, 223

Fan, Z., \& Chiueh, T. 2001, ApJ, 550, 547

Forni, O., \& Aghanim, N. 2004, A\&A, 420, 49

Freese, K., Adams, F. C., Frieman, J. A., \& Mottola, E. 1987, Nucl. Phys. B, 287, 797

Ge, J., Bechtold, J., \& Black, J. H. 1997, ApJ, 474, 67

Geisbüsch, J., Kneissl, R., \& Hobson, M. 2005, MNRAS, 360, 41

Itoh, N., Kohyama, Y., \& Nozawa, S. 1998, ApJ, 502, 7

Itoh, N., \& Nozawa, S. 2004, A\&A, 417, 827 
Kay, S. T., Liddle, A. R., \& Thomas, P. A. 2001, MNRAS, 325, 835

Kitayama, T., \& Suto, Y. 1996, ApJ, 469, 480

Lamarre, J. M., Puget, J. L., Bouchet, F., et al. 2003, New Astron. Rev., 47, 1017

Lima, J. A. S. 1996, Phys. Rev. D, 54, 2571

Lima, J. A. S., Silva, A. I., \& Viegas, S. M. 2000, MNRAS, 312, 747

Losecco, J. M., Mathews, G. J., \& Wang, Y. 2001, Phys. Rev. D, 64, 123002

Mather, J. C., Fixsen, D. J., Shafer, R. A., Mosier, C., \& Wilkinson, D. T. 1999, ApJ, 512, 511

Mennella, A., Bersanelli, M., Cappellini, B., et al. 2004, in Plasmas in the Laboratory and in the Universe: New Insights and New Challenges, AIP Conf. Proc., 703, 401

Mo, H. J., \& White, S. D. M. 2002, MNRAS, 336, 112

Molaro, P., Levshakov, S. A., Dessauges-Zavadsky, M., \& D'Odorico, S. 2002, A\&A, 381, L64

Navarro, J. F., Frenk, C. S., \& White, S. D. M. 1997, ApJ, 490, 493

Penzias, A. A., \& Wilson, R. W. 1965, ApJ, 142, 419
Puy, D. 2004, A\&A, 422, 1

Rephaeli, Y. 1980, ApJ, 241, 858

Rephaeli, Y. 1995, ARA\&A, 33, 541

Rephaeli, Y., \& Yankovitch, D. 1997, ApJ, 481, L55

Roth, K. C., Meyer, D. M., \& Hawkins, I. 1993, ApJ, 413, L67

Sazonov, S. Y., \& Sunyaev, R. A. 1998a, ApJ, 508, 1

Sazonov, S. Y., \& Sunyaev, R. A. 1998b, Astron. Lett., 24, 553

Sheth, R. K., \& Diaferio, A. 2001, MNRAS, 322, 901

Sheth, R. K., Mo, H. J., \& Tormen, G. 2001, MNRAS, 323, 1

Sheth, R. K., \& Tormen, G. 1999, MNRAS, 308, 119

Spergel, D. N., Verde, L., Peiris, H. V., et al. 2003, ApJS, 148, 175

Srianand, R., Petitjean, P., \& Ledoux, C. 2000, Nature, 408, 931

Sunyaev, R. A., \& Zeldovich, Y. B. 1972, Comments on Astrophys. Space Phys., 4, 173

Tauber, J. A. 2004, Adv. Space Res., 34, 491

Thaddeus, P. 1972, ARA\&A, 10, 305

Wright, E. L. 1979, ApJ, 232, 348

Zeldovich, Y. B., \& Sunyaev, R. A. 1969, Ap\&SS, 4, 301 